\title{
THE COHOMOLOGY RING OF A SMOOTH MANIFOLD $\left.{ }^{1}\right)$
}

\author{
BY \\ JAMES B. CARRELL
}

A fundamental result relating the topology of a smooth manifold and its global differential geometry is the theorem of De Rham. Let $\subseteq(X, R)$ denote the exterior algebra (over the reals $R$ ) of smooth differential forms on $X$, and let $\mathfrak{S}(X, R)$ denote its derived cohomology algebra. The De Rham theorem asserts that there is an algebra isomorphism of $\mathfrak{E}(X, R)$ with the singular cohomology algebra (cup product) $H(X, R)$ of $X$.

A generalization of this theorem to handle other coefficient domains is due to C. B. Allendoerfer and J. Eells, Jr. in [1]. For an arbitrary integral subdomain $A$ of $R$ they define a cochain complex $\mathfrak{\subseteq}(X, A)$ - the complex of $A$ pairs of forms on $X$ and prove that the derived cohomology module $\mathfrak{S}(X, A)$ of $\mathfrak{E}(X, A)$ is canonically isomorphic to the Čech cohomology module $\breve{H}(X, A)$ with values in $A$. This paper is a natural extension of the Allendoerfer-Eells paper.

Our concern is to define a ring structure for $\mathscr{S}(X, Z)$, where $Z$ denotes the integers, so that the canonical (module) isomorphism with $\breve{H}(X, Z)$ preserves products. The key is to define a certain cochain map $\mathfrak{s}(X, Z) \otimes \mathfrak{S}(Y, Z) \rightarrow \mathfrak{s}(X \times Y, Z)$, where $X$ and $Y$ are any smooth manifolds. This defines an exterior cup product $\mathfrak{S}(X, Z) \otimes \mathfrak{S}(Y, Z) \rightarrow \mathfrak{S}(X \times Y, Z)$ which sends $\xi \otimes \eta \in \mathfrak{S C}^{p}(X, Z) \otimes \mathfrak{S E}^{q}(Y, Z)$ to $\xi \times \eta \in \mathfrak{S e}^{p+q}(X \times Y, Z)$. Let $h_{1}: \mathfrak{S}(X, Z) \rightarrow \breve{H}(X, Z), h_{2}: \mathfrak{S}(Y, Z) \rightarrow \breve{H}(Y Z)$ and $h: \mathfrak{S}_{(}(X \times Y, Z) \rightarrow \check{H}(X \times Y, Z)$ be the canonical isomorphisms. Then a sheaf argument shows that $h(\xi \times \eta)=h_{1}(\xi) \times h_{2}(\eta)$ where $h_{1}(\xi) \times h_{2}(\eta)$ is the exterior cup product (or cartesian product) of $h_{1}(\xi)$ and $h_{2}(\eta)$ in the Cech theory. We define the interior cup product for $\mathfrak{S}(X, Z)$ via the homomorphism $\Delta^{*}: \mathfrak{S}(X \times X, Z) \rightarrow \mathfrak{S}(X, Z)$ induced by the diagonal map $\Delta: X \rightarrow X \times X$ and thus obtain a cohomology algebra $\mathfrak{S}_{\mathfrak{C}}(X, Z)$ isomorphic with the cohomology algebra $\breve{H}(X, Z)$. Finally we remark that the same method applies to arbitrary coefficient rings $A$ in $R$ to define an algebra $\mathfrak{S}(X, A)$ algebra isomorphic with $\check{H}(X, A)$.

The paper is organized as follows. The first section is devoted to a restatement of some of the definitions of [1]. In $\$ 2$ we discuss the general problem of introducing a product in $\mathfrak{S}(X, Z)$. In $\S 3$ we prove a lemma regarding general position of chains and singular sets which is needed in a later section. $\$ 4$ is devoted to proving the theorems stated in $\S 2$. In $\S 5$ we construct the exterior and interior cup products, and in $\S 6$ prove that the isomorphism extends to products. In $\S 7$ we make some remarks concerning products when $X$ is given a triangulation.

Received by the editors December 4, 1967.

( ${ }^{1}$ This work was partially supported by the U.S. Office of Naval Research under Contract Nonr 477 (15), Project NR 043186. 
This paper is essentially the author's doctoral dissertation written at the University of Washington under the direction of Carl B. Allendoerfer. The author wishes to acknowledge his indebtedness to Professor Allendoerfer for his continual help and encouragement. The author also wishes to thank Professor I. Namioka for his valuable assistance.

1. All manifolds $X, Y$, etc. considered in this paper will be smooth (i.e. $C^{\infty}$ ), paracompact, and connected. Let $\theta$ be a $p$-form defined and smooth on $X$ except for a subset $e(\theta)$ of $X$. We shall say that $e(\theta)$ is an allowable set of singularities, or briefly, $e(\theta)$ is allowable, if $e(\theta)$ is closed and there exists a locally finite family $\left\{N_{\alpha}\right\}$ of closed submanifolds of $X$ such that for each $\alpha, \operatorname{dim}\left(N_{\alpha}\right) \leqq \operatorname{dim}(X)-p-1$, and such that $e(\theta) \subset \bigcup\left\{N_{\alpha}\right\}$. This is a refinement of polyhedra in [1]. By a smooth singular $p$-cube on $X$ we shall mean a map $u: I^{p} \rightarrow X$ which is the restriction to $I^{p}$ of a smooth map of $R^{p}$ into $X$.

We will adhere to the notations of [1], $\S \S 2$ and 3, with the following exceptions. For $Z$ the integers, we define a $(Z, p)$ pair $(\theta, \omega)$ on $X$ as in [1], Definition 3A, except that we require that the singular sets $e(\theta)$ and $e(\omega)$ be allowable as defined above, and, for every smooth singular $p$-cube $u$ on $X$ admissible for $(\theta, \omega), R(\theta, \omega) u$ $=\int_{u} \theta-\int_{\partial u} \omega$ be an element of $Z$. We further make the following notational convention: if $(\theta, 0)$ is a $(Z, 0)$ pair on $X$ we agree that $e(0)=X$.

The following notations will be standard throughout. $(X, Z, p)$ will denote the additive semigroup of all $(Z, p)$ pairs on $X$, and $(X, R, p)$ will denote the additive semigroup of all $(R, p)$ pairs on $X$. $\mathfrak{\complement}^{p}(X)$ will denote the $Z$ module of equivalence classes of $(Z, p)$ pairs on $X$. An element of $\mathbb{E}^{p}(X)$ will be denoted by $[\theta, \omega]$ where $(\theta, \omega) \in(X, Z, p)$. $\Subset(X)=\sum_{p \geqq 0} \mathfrak{\complement}^{p}(X)$ will denote the complex of $Z$ pairs on $X$ with $d: \mathfrak{C}^{p}(X) \rightarrow \mathfrak{C}^{p+1}(X)$ defined by $d[\theta, \omega]=[0, \theta]$, and $\mathfrak{S}_{(}(X)=\sum_{p \geqq 0} \mathfrak{S}^{p}(X)$ will be the derived cohomology module of $\mathfrak{E}(X)$.

2. The key step in the construction of a product in $\mathfrak{S}(X)$ will be the construction of a certain cochain map $k: \mathfrak{\Im}(X) \otimes \mathfrak{\Im}(Y) \rightarrow \mathfrak{\Im}(X \times Y)$. In $\S 5$ we will exhibit a map $(X, R, p) \times(Y, R, q) \rightarrow(X \times Y, R, p+q)$ sending $(\theta, \omega) \in(X, R, p)$ and $(\psi, \eta)$ $\in(Y, R, q)$ into a pair denoted by $(\theta, \omega) \times(\psi, \eta)$. The properties of this map imply that $[(\theta, \omega) \times(\psi, \eta)]$ depends only on $[\theta, \omega]$ and $[\psi, \eta]$ and that $(\theta, \omega) \times(\psi, \eta)$ is a $Z$ pair if $(\theta, \omega)$ and $(\psi, \eta)$ are $Z$ pairs. This map will thus induce $k$.

More generally, suppose that for every $X, Y, p$, and $q$ we are given a map $(X, R, p) \times(Y, R, q) \rightarrow(X \times Y, R, p+q)$. We will show that if these maps satisfy certain natural requirements, which are in fact satisfied by the maps of $\$ 5$, then $(X, Z, p) \times(Y, Z, q)$ is mapped into $(X \times Y, Z, p+q)$, and the maps respect the equivalence classes. Such a class of maps will be called an exterior pairing of pairs.

Definition 2.1. An exterior pairing of pairs is a mapping $(X, R, p) \times(Y, R, q)$ $\rightarrow(X \times Y, R, p+q)$ defined for any two smooth manifolds $X$ and $Y$ and for any nonnegative integers $p$ and $q .(\theta, \omega) \times(\psi, \eta)$ will denote the pairing of $(\theta, \omega)$ $\in(X, R, p)$ and $(\psi, \eta) \in(Y, R, q)$. We require that the pairing satisfy: 
(1) if $(\alpha, \beta)=(\theta, \omega) \times(\psi, \eta)$, then $e(\alpha)=e(\omega) \times e(\psi) \cup e(\theta) \times e(\eta)$ and $e(\beta)=e(\omega)$ $\times e(\eta)$,

(2) $[(\theta, \omega) \times(\psi, \eta)]$ is bilinear in $(\theta, \omega)$ and $(\psi, \eta)$,

(3) (coboundary formula) if $(\theta, \omega) \in(X, R, p)$ then

$$
d[(\theta, \omega) \times(\psi, \eta)]=[(0, \theta) \times(\psi, \eta)]+(-1)^{p}[(\theta, \omega) \times(0, \psi)],
$$

and

(4) whenever $f: X^{\prime} \rightarrow X$ and $g: Y^{\prime} \rightarrow Y$ are smooth maps such that $\left(f^{*} \theta, f^{*} \omega\right)$ and $\left(g^{*} \psi, g^{*} \eta\right)$ are pairs, then

$$
\left[(f \times g)^{*}(\theta, \omega) \times(\psi, \eta)\right]=\left[\left(f^{*} \theta, f^{*} \omega\right) \times\left(g^{*} \psi, g^{*} \eta\right)\right] .
$$

TheOrem 2.2. Suppose an exterior pairing is given. Then for all $(\theta, \omega) \in(X, R, p)$ and for all $(\psi, \eta) \in(Y, R, q),[(\theta, \omega) \times(\psi, \eta)]$ depends only on $[\theta, \omega]$ and $[\psi, \eta]$.

THEOREM 2.3. Suppose an exterior pairing is given which maps $(X, Z, 0) \times(Y, Z, 0)$ into $(X \times Y, Z, 0)$ for all manifolds $X$ and $Y$. Then for all $p$ and $q,(X, Z, p) \times$ $(Y, Z, q)$ is mapped into $(X \times Y, Z, p+q)$.

The proofs of Theorems 2.2 and 2.3 will be deferred to $\S 4$. We may thus define $[\theta, \omega] \times[\psi, \eta]$ in an unambiguous way to obtain a cochain map $k: \mathfrak{s}(X) \otimes \mathfrak{S}(Y)$ $\rightarrow \mathfrak{E}(X \times Y)$ which is natural in an obvious sense. The exterior cup product defined by this pairing is the induced map $k: \mathfrak{S}(X) \otimes \mathfrak{S E}_{(}(Y) \rightarrow \mathfrak{F}(X \times Y)$. We will see in the proof of Theorem 2.3 that any two pairings which agree on $(X, Z, 0)$ $\times(Y, Z, 0)$ for any two manifolds $X$ and $Y$ determine the same map $k$.

There is a natural pairing of 0 pairs defined by setting $(\theta, 0) \times(\psi, 0)=(\theta \times \psi, 0)$, where $\theta \times \psi$ is the 0 -form on $X \times Y-(e(\theta) \times Y \cup X \times e(\psi))$ defined by $\theta \times \psi(x \times y)$ $=\theta(x) \cdot \psi(y)$. The pairings of $\S 5$ will extend this one. Note that we immediately obtain a commutative diagram

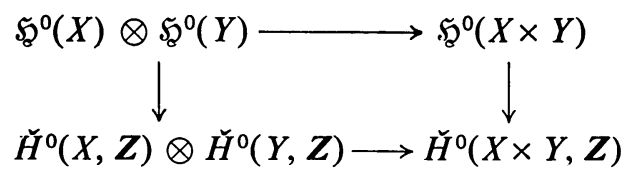

where the vertical maps are canonical isomorphisms.

We now discuss the problem of introducing cup products in $\mathfrak{F}_{(}(X)$ if we are given an exterior pairing. Let $(\theta, \omega) \in(X, Z, p)$ and $(\psi, \eta) \in(X, Z, q)$. Let $(\alpha, \beta)$ $\in(X \times X, Z, p+q)$ denote $(\theta, \omega) \times(\psi, \eta)$. If $\Delta: X \rightarrow X \times X$ is the diagonal map we may consider the singular forms $\Delta^{*} \alpha$ and $\Delta^{*} \beta$, where $e\left(\Delta^{*} \alpha\right)=e(\omega) \cap e(\psi)$ $\cup e(\theta) \cap e(\eta)$ and $e\left(\Delta^{*} \beta\right)=e(\omega) \cap e(\eta)$. If the singularities of $\Delta^{*} \alpha$ and $\Delta^{*} \beta$ are allowable, then we obtain a pair $\left(\Delta^{*} \alpha, \Delta^{*} \beta\right) \in(X, Z, p+q)$ which we denote by $(\theta, \omega) \vee(\psi, \eta)$. If $[\theta, \omega]$ and $[\psi, \eta]$ have representatives $(\theta, \omega)$ and $(\psi, \eta)$ so that $(\theta, \omega) \vee(\psi, \eta)$ is defined, we set $[\theta, \omega] \vee[\psi, \eta]=[(\theta, \omega)] \vee[(\psi, \eta)] .[\theta, \omega] \vee[\psi, \eta]$ is called the (interior) cup product of $[\theta, \omega]$ and $[\psi, \eta]$. Clearly, when everything is defined

$$
d([\theta, \omega] \vee[\psi, \eta])=d[\theta, \omega] \vee[\psi, \eta]+(-1)^{p}[\theta, \omega] \vee d[\psi, \eta]
$$


The crucial fact which allows us to define products in $\mathfrak{S}_{\mathfrak{C}}(X)$ is this: any two cohomology classes $\xi, \zeta \in \mathfrak{F}(X)$ are represented by equivalence classes $[\theta, \omega]$ and $[\psi, \eta]$ so that $[\theta, \omega] \vee[\psi, \eta]$ is defined. It is thus possible to define $\xi \vee \zeta$ to be the cohomology class of $[\theta, \omega] \vee[\psi, \eta]$. This gives rise to a commutative pairing $\mathfrak{S}(X) \otimes \mathfrak{S}(X) \rightarrow \mathfrak{E}(X)$.

3. Suppose $(\theta, \omega) \in(X, R, p)$ is a pair with the property that for every smooth singular $p$-cube $u$ on $X$ such that $u^{-1}[e(\theta)]$ is empty and $u^{-1}[e(\omega)]$ is a single point, $R(\theta, \omega) u$ is an element of $Z$. Then clearly $R(\theta, \omega) u \in Z$ for every admissible $u$ such that $u^{-1}[e(\omega)]$ is finite. Our next theorem shows that given a smooth singular cube admissible for $(\theta, \omega) \in(X, R, p)$, its admissible smooth homotopy class [1, Proposition 2D] contains a smooth singular cube $v$ such that $v^{-1}[e(\omega)]$ is indeed finite. Thus to show that $(\theta, \omega)$ is actually in $(X, Z, p)$ we need only show $R(\theta, \omega) u \in Z$ for every cube $u$ in general position with respect to $(\theta, \omega)$; that is, $u^{-1}[e(\theta)]=\varnothing$ and $u^{-1}[e(\omega)]$ is a singleton.

THEOREM 3.1. Let $(\theta, \omega) \in(X, R, p)$ with $p>0$. Then if $u$ is a smooth singular $p$-cube on $X$ admissible for $(\theta, \omega)$, there exists a smooth singular p-cube $v$ on $X$ admissible for $(\theta, \omega)$ such that

(1) $v^{-1}[e(\omega)]$ is finite,

(2) $v \simeq u$ through an admissible smooth homotopy, and

(3) $R(\theta, \omega) u=R(\theta, \omega) v$.

We need the following lemma which can be proved by induction on $k$ using Thom's Theorem on transverse regularity [3], [5].

LEMMA 3.2. Let $f: Y \rightarrow X$ be a smooth map, let $K$ be a compact subset of $Y$, and let $N_{1}, \ldots, N_{k}$ be closed submanifolds of $X$. Let $d$ be a metric on $X$ and let $\delta$ be a positive real number. Then there exists a smooth $g: Y \rightarrow X$ which is transverse regular to $N_{1}, \ldots, N_{k}$ on $K$ such that $d(g(y), f(y))<\delta$ if $y \in K$.

Proof of Theorem 3.1. Let $f: V \rightarrow X$ be a smooth extension of $u$ to an open neighborhood $V$ of $I^{p}$ in $R^{p}$. Suppose $\left\{N_{\alpha}\right\}$ is a locally finite collection of closed submanifolds of $X$ such that $e(\omega) \subset \bigcup\left\{N_{\alpha}\right\}$ and $\operatorname{dim}\left(N_{\alpha}\right) \leqq \operatorname{dim}(X)-p$ for each $\alpha$. Suppose $X$ has a Riemannian metric and let $d$ be the induced (distance) metric on $X$. Since $\left\{N_{\alpha}\right\}$ is locally finite and $f\left[I^{p}\right]$ is compact, $f\left[I^{p}\right]$ meets only finitely many $N_{\alpha}$, say $N_{\alpha_{1}}, \ldots, N_{\alpha_{k}}$. Choose a positive real number $\delta$ small enough so that if $g: V \rightarrow X$ is smooth and $d(g(t), f(t))<\delta$ for $t \in I^{p}$, then $g\left[I^{p}\right]$ meets only $N_{\alpha_{1}}, \ldots N_{\alpha_{k}}$, and $g \mid I^{p}$ is admissible for $(\theta, \omega)$. This is possible since $e(\theta), e(\omega)$, and

$$
\bigcup\left\{N_{\alpha}: \alpha \neq \alpha_{1}, \ldots, \alpha_{k}\right\}
$$

are closed. By Lemma 3.2 there exists such a $g$ which is transverse regular to $N_{\alpha_{1}}, \ldots, N_{\alpha_{k}}$ on $I^{p}$. Set $v=g \mid I^{p}$. Then by Corollary 10.8 of [4], taking $\delta$ sufficiently small, $v \simeq u$ by an admissible smooth homotopy, and hence (2) is proven. (3) follows at once from Proposition 2D of [1]. 
It remains to show that $v^{-1}[e(\omega)]$ is finite. We first note that $v^{-1}[e(\omega)]$ $\subset \bigcup v^{-1}\left[N_{\alpha_{i}}\right]$. Since $\operatorname{dim}\left(N_{\alpha_{i}}\right) \leqq \operatorname{dim}(X)-p$, since $g$ is transverse regular to $N_{\alpha_{i}}$ on $I^{p}$, and since $N_{\alpha_{i}}$ is closed, each $v^{-1}\left[N_{\alpha_{1}}\right]$ is a compact 0 dimensional submanifold of int $I^{p}$ and hence finite. This completes the proof of (1).

4. We assume now that an exterior pairing is given. Let $(\theta, \omega) \in(X, Z, p)$. If $U$ is an open subset of $X$ then the inclusion $i: U \rightarrow X$ induces a map $i^{*}:(X, Z, p)$ $\rightarrow(U, Z, p)$ which is just restriction. Let $\left(\theta_{U}, \omega_{U}\right)$ denote $i^{*}(\theta, \omega)$. Then $\theta_{U}$ and $\omega_{U}$ are the restrictions of $\theta$ and $\omega$ to $U$.

Let $U$ be a coordinate ball in $X$. If $p>0$ and if $e(\theta)=\varnothing$, then $d[\theta, \omega]=[0,0]$, and there exists by [1] a pair $(\pi, \mu) \in(U, Z, p-1)$ such that $d[\pi, \mu]=\left[\theta_{U}, \omega_{U}\right]$ as elements of $\mathfrak{C}^{p}(U)$. Furthermore $\pi=\omega_{U}-k \theta_{U}$ where $k \theta_{U}$ is the global $(p-1)$-form on $U$ of the Poincaré Lemma such that $d k \theta_{U}=\theta_{U}$.

LEMMA 4.1. Let $U$ be an open ball in $R_{n}$, and let $(\theta, \omega) \in(U, Z, p)$ with $p>0$ and $e(\theta)=\varnothing$. Let $(\psi, \eta) \in(Y, Z, q)$ be a pair such that $e(\psi)=\varnothing$. Then with $(\pi, \mu) \in$ $(U, Z, p-1)$ as above,

$$
d[(\pi, \mu) \times(\psi, \eta)]=[(\theta, \omega) \times(\psi, \eta)] .
$$

Proof. By the coboundary formula and the fact that $e(\psi)=\varnothing$,

$$
d[(\pi, \mu) \times(\psi, \eta)]=[(0, \pi) \times(\psi, \eta)]=[(0, \omega-k \theta) \times(\psi, \eta)] .
$$

Using bilinearity and the fact that $e(k \theta)=\varnothing$, we easily see that

$$
[(0, \omega-k \theta) \times(\psi, \eta)]=[(\theta, \omega) \times(\psi, \eta)],
$$

and hence the lemma follows.

Proof of Theorem 2.3. The proof will be by induction. The case $p=q=0$ is already assumed. Thus we assume that, for any smooth manifolds $X^{\prime}$ and $Y^{\prime}$, whenever $(\pi, \mu) \in\left(X^{\prime}, Z, p-1\right)(p>0)$ and $\left(\psi^{\prime}, 0\right) \in\left(Y^{\prime}, Z, 0\right)$, then $(\pi, \mu) \times\left(\psi^{\prime}, 0\right)$ $\in\left(X^{\prime} \times Y^{\prime}, Z, p-1\right)$.

Let $(\theta, \omega) \in(X, Z, p)$ and $(\psi, 0) \in(Y, Z, 0)$. We must show that $(\alpha, \beta)=(\theta, \omega)$ $\times(\psi, 0) \in(X \times Y, Z, p)$. To show this it suffices by $\S 3$ to show that $R(\alpha, \beta) u \in Z$ for any smooth singular $p$-cube $u$ on $X \times Y$ in general position with respect to the singularities of $(\alpha, \beta)$; i.e. $u^{-1}[e(\beta)]=$ point and $u^{-1}[e(\alpha)]=\varnothing$. Thus let $u$ be such a cube and set $x=u^{-1}[e(\beta)]$. Recall that $e(\alpha)=e(\theta) \times Y \cup e(\omega) \times e(\psi)$ and $e(\beta)$ $=e(\omega) \times Y$. Hence $u(x) \notin e(\theta) \times Y \cup X \times e(\psi)$ since $u(x) \in e(\omega) \times Y$. We may thus suppose, by [1], Proposition 2D, that $u\left[I^{p}\right] \subset U \times V$, where $U$ and $V$ are coordinate balls in $X$ and $Y$ such that $e(\theta) \cap U$ and $e(\psi) \cap V$ are both empty.

Let $(\pi, \mu) \in(U, Z, p-1)$ be the pair such that $d[\pi, \mu]=\left[\theta_{U}, \omega_{U}\right]$ as elements of $\mathfrak{S}^{p}(U)$ and $\pi=\omega_{U}-k \theta_{U}$. By naturality, the restriction of $[\alpha, \beta]$ to $U \times V$ is $\left[\left(\theta_{U}, \omega_{U}\right) \times\left(\psi_{V}, 0\right)\right]$. By Lemma 4.1, $d\left[(\pi, \mu) \times\left(\psi_{V}, 0\right)\right]=\left[\left(\theta_{U}, \omega_{U}\right) \times\left(\psi_{V}, 0\right)\right]$. Hence $R(\alpha, \beta) u=-R(\pi, \mu) \times\left(\psi_{V}, 0\right) \partial u$ is an element of $Z$. This completes the proof for $q=0$ and $p$ arbitrary. 
The proof is now completed by induction on $q$ with $p$ fixed but arbitrary, the first step of the induction having already been completed. It is clear how to proceed.

The proof of Theorem 2.2 is similar. By bilinearity it is obvious that we need only show $[(\theta, \omega) \times(\psi, \eta)]=[0,0]$ if either $[\theta, \omega]=[0,0]$ or $[\psi, \eta]=[0,0]$. The case $p=q=0$ is trivial and the induction step is proved by noting (with the above notation) that if $\left[\theta_{U}, \omega_{U}\right]=[0,0]$ and $e\left(\theta_{U}\right)$ is empty, then $[\pi, \mu]=[0,0]$.

5. We shall now construct an exterior pairing of pairs. We first give a motivating example.

Example. Suppose $X=R^{2}$ and suppose we are given pairs $(\theta, \omega)$ and $(\psi, \eta)$ on $X$ such that $\theta$ and $\psi$ are global 1 -forms, $e(\omega)$ is the $x$-axis, and $e(\eta)$ is the $y$-axis. We would like to find a smooth 1 -form $\beta$ with $e(\beta)=e(\omega) \cap e(\eta)$ and $d \beta=\theta \vee \psi$. We add the additional requirement that $\beta=-\theta \vee \eta$ near $e(\omega)-e(\omega) \cap e(\eta)$ and that $\beta=\omega \vee \psi$ near $e(\eta)-e(\omega) \cap e(\eta)$. Thus we need to define $\beta$ by patching. Let $\phi: X-e(\omega) \cap e(\eta) \rightarrow R$ be a smooth function such that $\phi=0$ near $e(\omega)-e(\omega) \cap e(\eta)$ and $\phi=1$ near $e(\eta)-e(\omega) \cap e(\eta)$. Then the form $\beta=-\theta \vee \eta+d(\phi \omega \vee \eta)$ satisfies our requirements. It can be shown that if $(\theta, \omega) \in(X, Z, 1)$ and $(\psi, \eta) \in(X, Z, 1)$, then $(\theta \vee \psi, \beta) \in(X, Z, 2)$. We will see that the pair $(\theta \vee \psi, \beta)$ may be taken for the definition of $(\theta, \omega) \vee(\psi, \eta)$.

Let $\pi_{1}: X \times Y \rightarrow X$ and $\pi_{2}: X \times Y \rightarrow Y$ be the projections. If $\theta$ is a $p$-form on $X$ and $\psi$ is a $q$-form on $Y$, we define a $(p+q)$-form $\theta \times \psi$ on $X \times Y$ by $\theta \times \psi=\pi_{1}^{*} \theta \vee \pi_{2}^{*} \psi$. $\theta \times \psi$ is called the cross product of $\theta$ and $\psi$. Clearly $d(\theta \times \psi)=(d \theta) \times \psi+(-1)^{p} \theta \times d \psi$. If $\theta$ is singular on $X$ with $e(\theta)$ allowable, then $\pi_{1}^{*} \theta$ is singular on $X \times Y$ with $e\left(\pi_{1}^{*} \theta\right)$ $=e(\theta) \times Y$ allowable. Thus if $(\theta, \omega) \in(X, R, p)(\operatorname{resp} .(X, Z, p))$, then $\left(\pi_{1}^{*} \theta, \pi_{1}^{*} \omega\right)$ $\in(X \times Y, R, p)$ (resp. $(X+Y, Z, p))$.

We now define the cross product of pairs. Let us first fix some notation. $(\theta, \omega)$ will always denote a fixed element of $(X, R, p)$ and $(\psi, \eta)$ will always denote a fixed element of $(Y, R, q)$. We set $M=X \times Y, M_{0}=M-e(\omega) \times e(\psi), M_{1}=M-e(\theta) \times e(\eta)$, and $M_{2}=M-e(\omega) \times e(\eta)$. If $p>0$ and $q>0$ we set $S=M-\left[e\left(\pi_{1}^{*} \omega\right) \cup e\left(\pi_{2}^{*} \psi\right)\right]$. $M_{0}, M_{1}, M_{2}$, and $S$ are dense open subsets of $M$.

We now define three $C^{\infty}$ functions as follows:

(1) Let $U_{0}, U_{1}$ be open subsets of $M_{0}$ such that $e\left(\pi_{1}^{*} \omega\right) \cap M_{0} \subset U_{0}, e\left(\pi_{2}^{*} \psi\right) \cap M_{0}$ $\subset U_{1}$ and $\bar{U}_{0} \cap \bar{U}_{1}=\varnothing$, the closures being taken in $M_{0}$. The $U_{i}$ exist since $e\left(\pi_{1}^{*} \omega\right)$ $\cap M_{0}$ and $e\left(\pi_{2}^{*} \psi\right) \cap M_{0}$ are closed disjoint subsets of $M_{0}$. If $p=0$, necessarily $U_{0}=M_{0}$ and $U_{1}=\varnothing$. If $e(\omega)=\varnothing$ we take $U_{0}=\varnothing$ and if $e(\psi)=\varnothing$ we take $U_{1}=\varnothing$. Let $\phi_{0}: M_{0} \rightarrow R$ be a $C^{\infty}$ function such that $\phi_{0}\left[U_{0}\right]=0$ and $\phi_{0}\left[U_{1}\right]=1$.

(2) Let $V_{0}, V_{1}$, be open subsets of $M_{1}$ such that $e\left(\pi_{1}^{*} \theta\right) \cap M_{1} \subset V_{0}, e\left(\pi_{2}^{*} \eta\right) \cap M_{1}$ $\subset V_{1}$, and $\bar{V}_{0} \cap \bar{V}_{1}=\varnothing$, the closures being taken in $M_{1}$. If $q=0$ we take $V_{0}=\varnothing$ and $V_{1}=M_{1}$. If $e(\theta)=\varnothing$ take $V_{0}=\varnothing$ and if $e(\eta)=\varnothing$ take $V_{1}=\varnothing$. Let $\phi_{1}: M_{1} \rightarrow R$ be a $C^{\infty}$ function such that $\phi_{1}\left[V_{0}\right]=0$ and $\phi_{1}\left[V_{1}\right]=1$.

(3) Let $W_{0}, W_{1}$ be open subsets of $M_{2}$ such that $e\left(\pi_{1}^{*} \omega\right) \cap M_{2} \subset W_{0}, e\left(\pi_{2}^{*} \eta\right)$ $\cap M_{2} \subset W_{1}$, and $\bar{W}_{0} \cap \bar{W}_{1}=\varnothing$, the closures being taken in $M_{2}$. Assume also that 
$W_{0} \subset U_{0}$ and $W_{1} \subset V_{1}$. If $p=0$ and $q>0, W_{0}=M_{2}$, and if $p>0$ and $q=0, W_{1}=M_{2}$. If $e(\omega)=\varnothing$ set $W_{0}=\varnothing$ and if $e(\eta)=\varnothing$ set $W_{1}=\varnothing$. Let $\phi_{2}: M_{2} \rightarrow R$ be a $C^{\infty}$ function such that $\phi_{2}\left[W_{0}\right]=0$ and $\phi_{2}\left[W_{1}\right]=1$.

Now if $p>0$ and $q>0$ define a smooth $(p+q-1)$-form $\bar{\beta}$ on $S$ by

$$
\bar{\beta}=-\phi_{0} \omega \times \psi+(-1)^{p} \phi_{1} \theta \times \eta+(-1)^{p-1} d\left(\phi_{2} \omega \times \eta\right) .
$$

If $p=0$ and $q>0$ define a smooth $(q-1)$-form $\bar{\beta}$ on $M-\left[e\left(\pi_{1}^{*} \theta\right) \cup e\left(\pi_{2}^{*} \eta\right)\right]$ by $\bar{\beta}=\phi_{1} \theta \times \eta$.

If $p>0$ and $q=0$ define a smooth $(p-1)$-form $\bar{\beta}$ on $M-\left[e\left(\pi_{1}^{*} \omega\right) \cup e\left(\pi_{2}^{*} \psi\right)\right]$ by $\bar{\beta}=\left(1-\phi_{0}\right) \omega \times \psi$.

If $p=q=0, \bar{\beta}$ is undefined.

TheOREM 5.1. Let $(\theta, \omega) \in(X, R, p)$ and $(\psi, \eta) \in(Y, R, q)$. If $p+q>0$ the form $\bar{\beta}$ has a unique smooth extension $\beta$ to $M_{2}=X \times Y-e(\omega) \times e(\eta)$, and $d \beta$ has a unique smooth extension $\alpha$ to $M_{0} \cap M_{1}=X \times Y-[e(\theta) \times e(\eta) \cup e(\omega) \times e(\psi)] .(\alpha, \beta)$ is thus an $(R, p+q)$ pair on $X \times Y$ with $e(\beta)=e(\omega) \times e(\eta)$ and $e(\alpha)=e(\theta) \times e(\eta) \cup e(\omega) \times e(\psi)$. If $p=q=0,(\theta \times \psi, 0)$ is an $(R, 0)$ pair on $X \times Y$ with $e(\theta \times \psi)=e(\theta) \times Y \cup X \times e(\psi)$.

Proof. We first note that any smooth extensions of $\bar{\beta}$ and $d \beta$ will be unique since their respective domains are dense subsets of $M$. We will carry out the proof for the case $p>0$ and $q>0$. The other cases are handled in a like manner. We want to show that $\bar{\beta}$ extends from $S$ to $M_{2}$. Now $M_{2}=S \cup W_{0} \cup W_{1}$ and since $W_{0} \cap W_{1}=\varnothing$, we need only show that $\bar{\beta}$ extends from $S \cap W_{i}$ to $W_{i}$ for $i=0,1$. Now $S \cap W_{0}$ $=W_{0}-e\left(\pi_{1}^{*} \omega\right)$, and since $W_{0} \subset \phi_{0}^{-1}[0] \cap \phi_{2}^{-1}[0], \quad \beta=(-1)^{p} \phi_{1} \theta \times \eta$ on $S \cap W_{0}$. Since $W_{0}$ is disjoint from $e\left(\pi_{2}^{*} \eta\right),(-1)^{p} \phi_{1} \theta \times \eta$ extends immediately to $W_{0}-e\left(\pi_{1}^{*} \theta\right)$. But $W_{0} \subset M_{1}$ so $\phi_{1}=0$ near $W_{0} \cap e\left(\pi_{1}^{*} \theta\right)$, and hence $\bar{\beta}$ extends smoothly to $W_{0}$. The extension of $\bar{\beta}$ to $W_{1}$ is similar.

We next show that $d \beta$ has a smooth extension $\alpha$ to $M_{0} \cap M_{1}$. On $S$,

$$
\begin{aligned}
d \beta= & (-1)^{p} d\left(\phi_{1} \theta \times \eta\right)-d\left(\phi_{0} \omega \times \psi\right) \\
= & \left(\phi_{1}-\phi_{0}\right) \theta \times \psi+(-1)^{p} \pi_{1}^{*} \omega \vee d\left(\phi_{0} \pi_{2}^{*} \psi\right) \\
& +(-1)^{p} d\left(\phi_{1} \pi_{1}^{*} \theta\right) \vee \pi_{2}^{*} \eta .
\end{aligned}
$$

As before we can show that $\pi_{1}^{*} \omega \vee d\left(\phi_{0} \pi_{2}^{*} \psi\right)$ extends smoothly to $M_{0}, d\left(\phi_{1} \pi_{1}^{*} \theta\right) \vee \pi_{2}^{*} \eta$ extends smoothly to $M_{1}$, and $\left(\phi_{1}-\phi_{0}\right) \theta \times \psi$ extends smoothly to $M_{0} \cap M_{1}$. Thus $\alpha$ is defined and smooth where asserted.

Definition 5.2. We denote by $(\theta, \omega) \times(\psi, \eta)$ the $(R, p+q)$ pair obtained in Theorem 5.1 from $(\theta, \omega) \in(X, R, p)$ and $(\psi, \eta) \in(Y, R, q)$ by patching. $(\theta, \omega) \times(\psi, \eta)$ is called the cross product of $(\theta, \omega)$ and $(\psi, \eta)$.

REMARK. For different choices of neighborhoods $U_{i}^{\prime}, V_{i}^{\prime}, W_{i}^{\prime}$ and functions $\phi_{j}^{\prime}$ satisfying (1), (2), (3) we may construct another $(R, p+q)$ pair $\left(\alpha^{\prime}, \beta^{\prime}\right)$ on $X \times Y$ from $(\theta, \omega)$ and $(\psi, \eta)$ by patching. A close scrutiny of the form $\beta-\beta^{\prime}$ shows that all its singularities are removable and thus $\left[\alpha^{\prime}, \beta^{\prime}\right]=[\alpha, \beta]$. We conclude that $[\alpha, \beta]$ is independent of the choices of patching functions $\phi_{0}, \phi_{1}, \phi_{2}$. 
THEOREM 5.3. The assignment of $(\theta, \omega) \in(X, R, p)$ and $(\psi, \eta) \in(Y, R, q)$ to $(\theta, \omega) \times(\psi, \eta)$ determines an exterior pairing.

The proof of Theorem 5.3 is straightforward and will be omitted.

We have thus shown the existence of an exterior pairing extending the natural pairing of 0 pairs discussed in $\S 2$. We thus have, by $\S 2$, homomorphisms

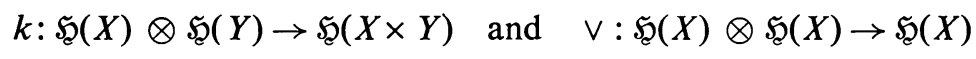

which define the exterior and interior cup products for $\mathfrak{S}(X)$.

6. We shall now show that $\mathfrak{S}(X)$ and $\breve{H}(X, Z)$ are isomorphic as rings. The complex $\mathfrak{\subseteq}(X)$ determines in a natural way a presheaf of complexes on $X$ : namely we assign to an open subset $U$ of $X$ the complex $\Subset(U)$. If $V \subset U$, the restriction $\mathfrak{S}(U) \rightarrow \mathfrak{C}(V)$ is induced by the inclusion $i: V \rightarrow U$. Obviously the restrictions commute with $d$. The differential graded sheaf associated with this presheaf is denoted by $\mathscr{C}_{X}=\sum_{p \geqq 0} \mathscr{C}_{X}^{p}$. By [1] we have a resolution of $Z, 0 \rightarrow Z \rightarrow \mathscr{C}_{X}^{0} \rightarrow \mathscr{C}_{X}^{1}$ $\rightarrow \cdots$. Let $Y$ be another manifold. Then for $U$ open in $X$ and $V$ open in $Y$ we have by $\$ 5$ homomorphisms

$$
\mathfrak{\mho}^{p}(U) \otimes \mathfrak{\mho}^{q}(V) \rightarrow \mathfrak{\mho}^{p+q}(U \times V)
$$

commuting with the restrictions. These give rise to a homomorphism of differential sheaves $\mathscr{C}_{X} \otimes \mathscr{C}_{Y} \rightarrow \mathscr{C}_{X \times Y}$. This homomorphism is compatible with the canonical homomorphism $\boldsymbol{Z} \otimes \boldsymbol{Z} \rightarrow \boldsymbol{Z}$; i.e.

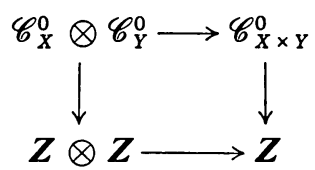

commutes. It follows from [2, II, 6.2] that we have a commutative diagram

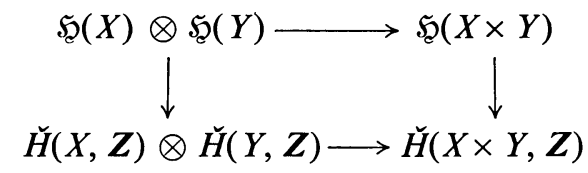

where the horizontal maps are defined by exterior cup products and the vertical maps are canonical isomorphisms.

From the definition of the (interior) cup products for $\mathfrak{S}_{(}(X)$ we deduce

THEOREM 6.1. Let $\mathfrak{S E}(X)$ have the graded ring structure induced by the cup product. Then the canonical (module) isomorphism $\mathfrak{S}(X) \rightarrow \breve{H}(X, Z)$ is an isomorphism of graded rings.

7. We now make some remarks on products when $X$ is given a triangulation. These may be applied to give an alternate proof of Theorem 6.1. Our first theorem shows that the cross product of pairs ( $\$ 5)$ behaves like the cross product of ordinary cochains. 
TheOrem 7.1. Let $(\theta, \omega) \in(X, R, p)$ and $(\psi, \eta) \in(Y, R, q)$. Let $u$ (resp. $v)$ be a smooth singular cube on $X$ (resp. $Y)$ admissible for $(\theta, \omega)($ resp. $(\psi, \eta))$. Then

$$
R(\theta, \omega) \times(\psi, \eta) u \times v=R(\theta, \omega) u \cdot R(\psi, \eta) v .
$$

Proof. Obviously $u \times v$ is admissible for $(\theta, \omega) \times(\psi, \eta)$. Set $(\alpha, \beta)=(\theta, \omega) \times(\psi, \eta)$. If $p>0$ and $q>0$ we have

$$
\beta=-\phi_{0} \omega \times \psi+(-1)^{p} \phi_{1} \theta \times \eta+(-1)^{p-1} d\left(\phi_{2} \omega \times \eta\right),
$$

where $\phi_{0}, \phi_{1}, \phi_{2}$ are certain functions defined in $\S 5$. We may alter the choice of the $\phi_{i}$ without changing $[\alpha, \beta]$. Hence we assert:

(1) $\phi_{0}$ may be chosen so that $|u \times v| \subset$ int $\phi_{0}^{-1}[0]$,

(2) $\phi_{1}$ may be chosen so that $|u \times v| \subset$ int $\phi_{1}^{-1}[1]$, and

(3) $\phi_{2}$ may be chosen so that $|(\partial u) \times v| \subset$ int $\phi_{2}^{-1}[1]$.

Thus near $|u \times v|, \alpha=\theta \times \psi$ and $\beta=(-1)^{p} \theta \times \eta+(-1)^{p-1} d\left(\phi_{2} \omega \times \eta\right)$. We also note that

$$
e\left(\phi_{2} \omega \times \eta\right) \cap|u \times(\partial v)|=\varnothing .
$$

Hence

$$
\begin{aligned}
\int_{u \times v} \alpha-\int_{\partial(u \times v)} \beta & =\int_{u \times v} \theta \times \psi-\int_{(\partial u) \times v} \omega \times \psi-\int_{u \times(\partial v)} \theta \times \eta+\int_{u \times(\partial v)} d\left(\phi_{2} \omega \times \eta\right) \\
& =\int_{u \times v} \theta \times \psi-\int_{(\partial u) \times v} \omega \times \psi-\int_{u \times(\partial v)} \theta \times \eta+\int_{(\partial u) \times(\partial v)} \omega \times \eta
\end{aligned}
$$

by Stokes' Theorem and (3).

Since $\int_{u \times v} \theta \times \psi=\int_{u} \theta \cdot \int_{v} \psi$ etc., we have, therefore,

$$
R(\alpha, \beta) u \times v=R(\theta, \omega) u \cdot R(\psi, \eta) v .
$$

Now suppose $X=M$ and $Y=N$ are (smooth) cubical complexes and let $M_{*}$ and $N_{*}$ denote their dual cell complexes, which are again cubical complexes. $M \times N$ is a cubical complex in a natural way with $(M \times N)_{*}=M_{*} \times N_{*}$. Let $\subseteq(M)$ and $\subseteq(N)$ be the cochain complexes defined in [1], §5. Then the cross product defines a cochain map $\mathfrak{\subseteq}(M) \otimes \mathfrak{C}(N) \rightarrow \mathfrak{\subseteq}(M \times N)$.

Let $C(M)$ and $C(N)$ denote the ordinary complexes of smooth cubical cochains on $M$ and $N$ respectively. Then there exists an isomorphism $h: \mathfrak{s}(M) \rightarrow C(M)$ given by $h([\theta, \omega]) c=R[\theta, \omega] c$ for any smooth cubical chain $c$ on $M$. Combining these facts with the fact that the inclusion $\subseteq(M) \rightarrow \circlearrowleft(X)$ induces an isomorphism of cohomology rings, we obtain from Theorem 7.1

THEOREM 7.2. Let $X$ and $Y$ have smooth cubical triangulations $M$ and $N$ respectively. Let $H(M, Z), H(N, Z)$, and $H(M \times N, Z)$ denote the cubical (or singular cubical) cohomology rings of $M$ and $N$ respectively with values in $Z$. Then there exists a commutative diagram

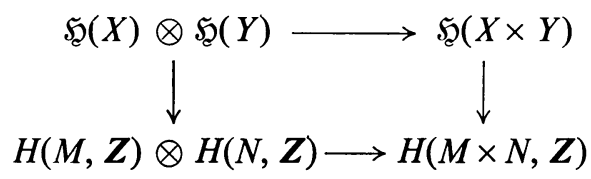


where the vertical arrows represent the isomorphisms defined above and the horizontal arrows represent the exterior cup products.

A detailed discussion involving the notion of a diagonal approximation can be given to establish the equivalence of $\mathfrak{S}_{\mathfrak{g}}(X)$ and $H(X, Z)$ (singular cohomology) as rings.

\section{BIBLIOGRAPHY}

1. C. B. Allendoerfer and J. Eells, Jr., On the cohomology of smooth manifolds, Comment. Math. Helv. 32 (1958), 165-179.

2. R. Godement, Théorie des faisceaux, Hermann, Paris, 1958.

3. J. Milnor, Differential topology, mimeographed notes, Princeton Univ., Princeton, N. J., 1958.

4. —_, Morse theory, Princeton Univ. Press, Princeton, N. J., 1963.

5. S. Sternberg, Lectures on differential geometry, Prentice-Hall, Englewood Cliffs, N. J., 1964.

6. H. Whitney, Geometric methods in cohomology theory, Proc. Nat. Acad. Sci. U.S.A. 33 (1947), 7-9.

UNIVERSITY OF WASHINGTON,

Seattle, Washington 